\title{
Endoscopic and Robotic Stapes Surgery: Review with Emphasis on Recent Surgical Refinements
}

\author{
Sean C. Sheppard ${ }^{1}$. Marco D. Caversaccio ${ }^{1} \cdot$ Lukas Anschuetz $^{1}$ (1)
}

Accepted: 1 December 2021 / Published online: 6 January 2022

(c) The Author(s) 2022

\begin{abstract}
Purpose of Review Stapes surgery has been established as the gold standard for surgical treatment of conductive hearing loss in otosclerosis. Excellent outcomes with very low complication rate are reported for this surgery. Recent advances to improve surgical outcome have modified the surgical technique with endoscopes, and recent studies report development of robotical assistance. This article reviews the use of endoscopes and robotical assistance for stapes surgery.

Recent Findings While different robotic models have been developed, 2 models for stapes surgery have been used in the clinical setting. These can be used concomitant to an endoscope or microscope. Endoscopes are used on a regular base regarding stapes surgery with similar outcomes as microscopes. Endoscopic stapes surgery shows similar audiological results to microscopic technique with an advantage of less postoperative dysgeusia and pain. Its utility in cases of revision surgery or malformation is emphasized.

Summary Endoscopic stapes surgery is used on a regular basis with excellent outcomes similar to the microscopic approach, while reducing surgical morbidity. Robotic technology is increasingly being developed in the experimental setting, and first applications are reported in its clinical use.
\end{abstract}

Keywords Stapes surgery · Robot-assisted stapedotomy $\cdot$ Endoscopic stapedotomy · Otosclerosis $\cdot$ Middle ear surgery Minimal-invasive surgery

\section{Introduction}

Stapes surgery has been widely established as the gold standard for treatment of conductive hearing loss in otosclerosis. The procedure requires a high degree of precision and experience by the performing ear surgeon. A high successrate, defined by a postoperative air-bone gap of less than 10

This article is part of the Topical Collection on OTOLOGY: Otosclerosis and Stapes Surgery

Lukas Anschuetz

anschuetz.lukas@gmail.com

Sean C. Sheppard

sean.sheppard@insel.ch

Marco D. Caversaccio

marco.caversaccio@insel.ch

1 Department of Otorhinolaryngology, Head and Neck Surgery, Inselspital, Bern University Hospital, and University of Bern, Bern, Switzerland
$\mathrm{dB}$ and low complication rates are described in the literature [1]. Although rare, postoperative complications include vertigo as well as a worsened auditory threshold. Furthermore, a risk of irreversible partial or complete sensorineural hearing loss is reported in $0.7-3 \%$ of patients [2]. Alternatively, the hearing loss may be addressed using hearing aids, which should always be proposed during patient counseling [3].

Rosen introduced the surgical treatment in 1952 using an operating microscope [1]. Since then, surgical steps of stapedectomy/stapedotomy have been constant: raising the tympanomeatal flap, lowering the scutum, sectioning the posterior tendon and crura, footplate fenestration, piston placement and crimping, and repositioning the tympanomeatal flap. Surgical difficulties regarding stapes surgery include a small and narrow surgical and visual field. Moreover, even small amounts of bleeding can reduce the visual field impeding the safe and efficient continuation of the operation. Additionally, important anatomical structures close to the oval window, such as the facial nerve, the chorda tympani and ossicles are at risk of iatrogenic damage. To improve the outcome and decrease risk 
of complications, technical modifications were proposed. The most difficult steps requiring extreme precision are the fenestration of the stapes footplate and crimping of the prosthesis. Stapedotomy has shown to have a similar outcome compared to stapedectomy in creating a small fenestra with a mechanical drill or laser $[4,5]$. Many different prostheses with differences in material, size, and shape have been developed, including the option of a self-crimping prosthesis [6, 7]. Furthermore, different kinds of lasers have been used increasingly to overcome limitations of mechanical footplate fenestration [8]. It has been well documented that a significant difference of outcome was reported in expert ear surgeons compared to trainees [9].

Endoscopic ear surgery (EES) has gained increasing popularity for all kinds of middle ear surgery in the past years. The main advantage of endoscopes is a better field of view with the possibility of using angled lenses. Regarding stapes surgery, similar outcomes as compared to the microscopic approach have been reported [10].

Robotic surgery nowadays has reached all surgical specialties including middle ear surgery. Its interest relies in exceeding the physical abilities limited by the human hand for improved movement and force accuracy. In the field of microsurgery, such as ophthalmology, neurosurgery, and reconstructive surgery, it has gained increasing attention. Even in experienced surgeons, involuntary gestures such as tremor, jerking, and drifting can occur. Robots have the ability to inhibit these involuntary gestures, resulting in more microsurgical precision, accuracy, and reducing forces applied on other structures [2].

\section{Methods}

A comprehensive literature search was conducted on PubMed with the following terms: endoscopic stapes, endoscopic stapedotomy, endoscopic stapedectomy, robotic stapes, robotic stapedotomy, robotic stapedectomy (date of last access: 22.07.2021). Out of 189 screened articles, we selected two types of articles. For endoscopic stapes surgery, only metaanalyses were considered, with a yield of 5 studies. A total of 16 studies were selected for robotic surgery focusing on stapes surgery. We included reports with robots used for experimental and/or clinical use in stapes surgery. One study reported a middle ear surgical robot with clinical application for chronic otitis. Corresponding authors were not contacted. Stapes surgery outcome was measured in means of time, surgical success, and complication rates.

\section{Review and Discussion}

\section{Endoscopic Stapes Surgery}

EES is becoming more prevalent as a valuable minimalinvasive approach for middle ear surgery. First reported as an adjunct to microscopic surgery, it is now widely used as the main tool for procedures such as tympanoplasty, ossiculoplasty, cholesteatoma resection, and stapes surgery. Fully endoscopic procedures are referred to as "transcanal endoscopic ear surgery" [11], avoiding the need of an external incision (retroauricular, endaural). The microscope remains the appropriate approach for mastoidectomy and the access to the antrum.

Concerning ESS, we reviewed 5 meta-analyses published between 2018 and 2020 comparing the endoscopic vs. microscopic approach as reported in Table 1. The primary outcome of all studies was postoperative auditory gain, usually considered with an airbone-gap of less than $10 \mathrm{~dB}$. The meta-analysis of Fang et al. [12] showed a favorable odds ratio of 1.99 for ESS while other metaanalyses did not show a statistically significant difference. Bone removal, when analyzed, was shown to be significantly reduced in ESS compared to microscopic stapes surgery (MSS) [12, 13]. While Ho et al. [14] reported no differences in manipulation of the chorda tympani in both groups, all other studies show a significant lower injury risk. When analyzed, all studies show lower postoperative dysgeusia in ESS. For secondary outcomes, patientreported postoperative pain was reduced in the ESS compared to the MSS group in two out of three studies [12, $15 \bullet \bullet]$. Postoperative dizziness and operative time did not show differences in the groups. Other complications were not shown as significant.

Regarding these meta-analyses, endoscopic or microscopic approach for stapes surgery appears to report a similar outcome in terms of postoperative airbone-gap. ESS has the advantage of being a minimal-invasive approach which often requires less bone removal and could result in a reduction of postoperative pain. However, the transcanal microscopic approach using an ear speculum can be considered equal in this regard [16]. As a limitation, measures of postoperative dizziness and pain were reported in different scales between the studies. Subjective symptoms are furthermore influenced by cultural factors and could add a potential bias $[15 \bullet \bullet]$. Overall, we must note that most included studies have a retrospective setting and a small sample size. Only 2 randomized controlled studies have been published until now. Audiological outcome can also be biased by use of different kinds and sizes of piston prosthesis [17].

In addition, ESS seems to reduce the risk of chorda tympani injury and consequently postoperative dysgeusia. The benefit of the wide-angled view of the endoscope allows a better visualization which aids in avoiding excessive manipulation of the chorda tympani and potential injury. All middle ear structures are reported to be better viewed endoscopically except for the antrum. The stapes footplate region with its narrow important structures as the facial nerve or ossicles is well seen through the endoscope. 


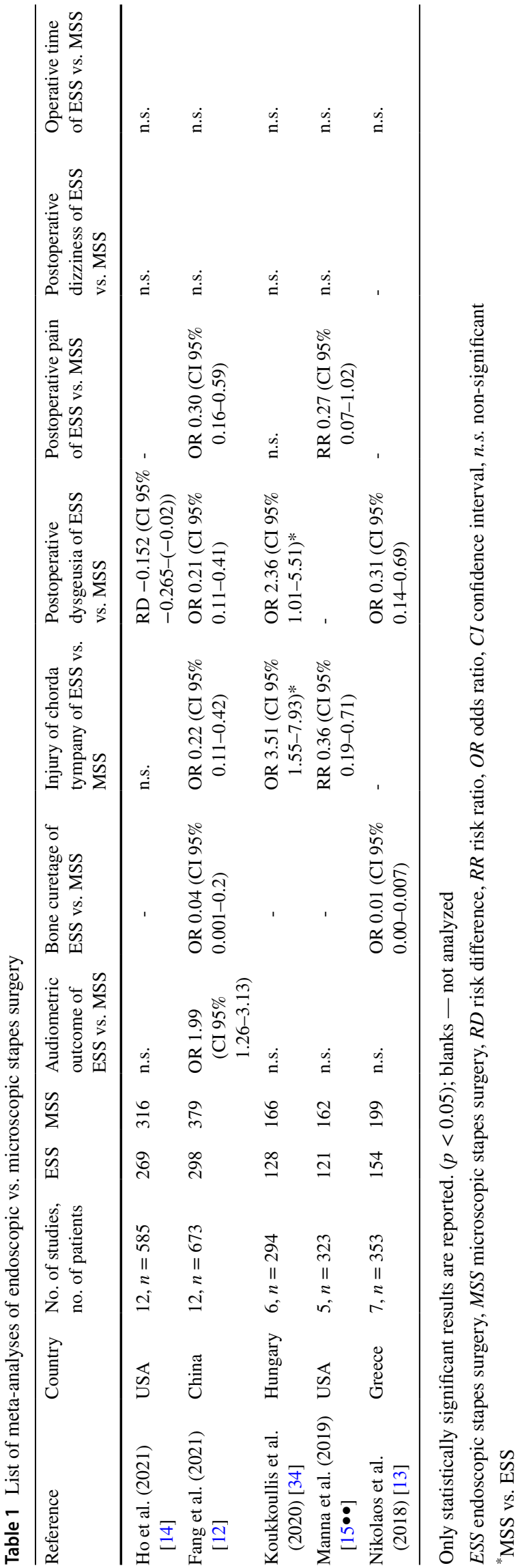

Equipment required for endoscopic surgery consists of a light source, rigid endoscopes (usually 3-mm diameter, $15-\mathrm{cm}$ length, $0^{\circ}$ and $45^{\circ}$ angles), a high definition camera, and video screen. The angles of the endoscopes vary depending on surgeons' preferences and patients' anatomy. A $45^{\circ}$ or $70^{\circ}$ angled-endoscope permits view of hidden recesses such as retrotympanic areas [18]. Advances in imaging technology (e.g., 4K) permit a high-definition image permitting a wide-angled view and enhanced magnification. A better view of the ossicle chain as well as the stapes is beneficial during revision surgery or to detect malformations [11]. While the wide-angled view is a main advantage of the endoscope, small amounts of bleeding can quickly obstruct the field of view and require recurrent cleaning of the scope [19]. To address this issue, the use of cottonoids soaked with epinephrine (1:1000) has shown to provide adequate bleeding control. A further challenge of endoscopic surgery is to rely on a single-handed technique, where the non-dominant hand holds the endoscope. A 2-handed technique has been tried with an endoscope holder or with the help of a robotic arm to hold the endoscope [20, 21•].

Lastly, authors often report the loss of perceptions of depth as an issue in endoscopic surgery. To address this issue, 3D endoscopes have been developed. In our experience, the use of a 3D endoscope did not change the surgical outcome and relies mostly on the surgeon's preference [22]. Operative time mainly depends on the experience of the operating surgeon. Across the literature, no significant difference in operative time between microscopic and endoscopic procedures was reported.

Finally, since EES has been developed at a later stage than microscopic surgery, the learning curve is often reported to be longer. For example, a number of 60 procedures has been reported for a microscopically trained surgeon to change his technique to endoscopic surgery for tympanoplasty [23]. This issue may be overcome by pre-clinical training using an ovine ex vivo model for training EES. The initial model for EES was further refined and validated regarding stapes surgery including the use of laser and has also been shown to be suitable for advanced and salvage procedures [24-26].

\section{Robotic Stapes Surgery}

Three types of robots have been reported for stapes surgery: a comanipulated system where the surgeon and the robot hold the tool conjointly; a teleoperated system where the surgeon controls the arm from a distance; and a semiautonomous system where the robot executes a surgical step, passively or actively guided [3]. A summary of robots used in stapes surgery is shown in Table 2. For each robot, all clinical studies and the latest experimental studies were included.

Comanipulated systems have only been used in the experimental setting for stapes footplate fenestration and 
placement of a piston prosthesis. Robotical assistance with "Steady Hand" was used for stapes fenestration with a micropick, resulting in reduced force applied to the stapes footplate and improved accuracy for less experienced surgeons [27]. Although with no statistical significance, "Micron" was reported to reduce tremor and accuracy of the fenestration with $\mathrm{CO}_{2}$ laser [28]. "REMS" was developed to reduce maximum force applied to the incus for prosthesis placement and crimping [29]. One of the main advantages of a comanipulated system is their ease of use, preserving tactile feedback with the ability to reduce tremor, measure force, and avoid involuntary gestures. Less experienced surgeons profited more of robotical assistance than experts.

Teleoperated robot-based systems for middle ear surgery have mostly been reported to execute stapes fenestration or crimping of the prosthesis. Out of the different systems, two devices have been used in the clinical setting. "RobOtol" was designed for middle ear surgery, and particularly for stapes surgery [30]. It is the only device reported to execute all tasks of the operation. Recently, Vittoria et al. published a clinical report of 32 patients successfully operated on with the "RobOtol" as an endoscope or micro-instrument holder [21•]. The robot assistance was used as a laser holder to vaporize the stapedial tendon, posterior and anterior crus and fenestration of the footplate. 9 patients with otosclerosis were successfully treated resulting in a 1-month postoperative airbone gap of $10+-1 \mathrm{~dB}$ and no intra or postoperative complications. Chorda tympani was preserved in all subjects, with no vertigo, tinnitus, or facial palsy. Therefore, it showed to be a safe device, with a quick learning curve for application. Time of setup of the robot was similar to the microscope; however, operating time was not reported.

The MMS- 2 was tested for stapedotomy and prosthesis placement in chronic otitis but had the disadvantage of an insufficient number of degrees of freedom to perform all middle ear procedures [31]. Furthermore, sterilization was an issue. Although developed for stapedotomy, MMS-2 was only used in the clinical setting for chronic otitis through placement of TORP and PORP prostheses [32]. To our knowledge, it has not been used for clinical stapes surgery. Although being less user-friendly, teleoperated systems achieve more tremor suppression, improve motion control accuracy and motion scaling with a broader angle of movement.

One autonomous-semiautonomous system was developed as an automatic micro-drill for the stapes footplate with an accuracy of up to $0.02 \mathrm{~mm}$. In our experience, we successfully developed and used an autonomous robotic system for middle ear access for cochlear implantation in 9 patients [33]. While the procedure was safely done, it was more timeconsuming and labor-intensive. Further developments for refinements and optimization of work flow are ongoing.

Although still in a mostly laboratory setting, recent advances and a few clinical studies show growth and advances in robotics for middle ear surgery. Technical tasks could be performed with robot assistance increasing the accuracy of movement and higher control of force. Moreover, these robotic assistances usually take up a lot of volume in the confined space of the operating room. While their use shows a growing interest, there are still limited data in the clinical application with no proof of reduced rates of complications or better surgical outcome. In the field of endoscopic sinus surgery, the "iArmS" robotic armrest has been developed and used in the clinical setting. It was reported to reduce the lens cleaning interval potentially reducing operating time [20]. Research and development and use of robotic assistance require financial investments with costly devices. Actually, no clear advantage of robotic surgery showed a better outcome of a reduced operating time [3].

Table 2 List of reported robots for stapes surgery

\begin{tabular}{|c|c|c|c|c|c|}
\hline Robot type & Reference & Country & Robot name & Setting & Tasks \\
\hline \multirow[t]{3}{*}{ Comanipulated } & Razavi et al. (2019) [29] & USA & REMS & Experimental & Prost,Crimp \\
\hline & Vendrametto et al. (2015) [28] & Italy & Micron & Experimental & Stap \\
\hline & Rothbaum et al. (2002) [27] & USA & Steady Hand & Experimental & Stap \\
\hline \multirow[t]{7}{*}{ Teleoperated } & Vittoria et al. (2021) [21•] & France & RobOtol & Clinical & All \\
\hline & Miroir et al. (2012) [30] & France & RobOtol & Experimental & All \\
\hline & Strauss et al. (2012) [32] & Germany & MMS-2 & Clinical & Prost* \\
\hline & Peschka et al. (2015) [31] & Germany & MMS-2 & Experimental & Stap \\
\hline & Yasin et al. (2016) [35] & USA & Prototype custom & Experimental & Prost \\
\hline & Bell et al. (2012) [36] & Switzerland & Robotics Department NTB & Experimental & Crimp \\
\hline & Runge et al. (2011) [37] & Germany & TUM Mikromanipulator & Experimental & Stap \\
\hline Semiautonomous & Brett et al. (1995) [38] & United Kingdom & Automatic Micro-drill & Experimental & Stap \\
\hline
\end{tabular}

TMF tympanomeatal flap, Scut lowering of the scutum, Crur sectioning of tendon and crura, Stap stapedotomy, Prost prosthesis placement, Crimp crimping

*PORP and TORP prosthesis were placed for chronic ear infections 


\section{Conclusion}

While the surgical steps of stapedotomy/stapedectomy have not changed, new tools are being developed to improve the safety and outcome of the procedure. Endoscopic ear surgery is increasingly applied and reaches to stapes surgery too. Reported outcome is similar to a microscopic approach, with an advantage of less postoperative dysgeusia and pain. Robotical assistance is under development for application in the clinical setting. Further studies in a prospective setting are needed to evaluate if a surgical technique is superior to the other.

Funding Open access funding provided by University of Bern.

\section{Compliance with Ethical Standards}

Conflict of Interest LA is a consultant for Stryker ENT. The other authors declare that they have no conflict of interest.

Human and Animal Rights and Informed Consent This review article does not contain any studies with human or animal subjects performed by any of the authors.

Open Access This article is licensed under a Creative Commons Attribution 4.0 International License, which permits use, sharing, adaptation, distribution and reproduction in any medium or format, as long as you give appropriate credit to the original author(s) and the source, provide a link to the Creative Commons licence, and indicate if changes were made. The images or other third party material in this article are included in the article's Creative Commons licence, unless indicated otherwise in a credit line to the material. If material is not included in the article's Creative Commons licence and your intended use is not permitted by statutory regulation or exceeds the permitted use, you will need to obtain permission directly from the copyright holder. To view a copy of this licence, visit http://creativecommons.org/licenses/by/4.0/.

\section{References}

Papers of particular interest, published recently, have been highlighted as:

- Of importance

$\bullet$ Of major importance

1. Rosen S. Palpation of stapes for fixation; preliminary procedure to determine fenestration suitability in otosclerosis. AMA Arch Otolaryngol. 1952;56:610-5.

2. Daoudi H, Torres R, Mazalaigue S, Sterkers O, Ferrary E, Nguyen Y. Analysis of forces during robot-assisted and manual manipulations of mobile and fixed footplate in temporal bone specimens. Eur Arch Otorhinolaryngol. 2021. https://doi.org/ 10.1007/s00405-020-06553-z.

3. Nguyen Y, Bernardeschi D, Sterkers O. Potential of robot-based surgery for otosclerosis surgery. Otolaryngol Clin North Am. 2018;51:475-85.
4. Sedwick JD, Louden CL, Shelton C. Stapedectomy vs stapedotomy. Do you really need a laser? Arch Otolaryngol Head Neck Surg. 1997;123:177-80.

5. House HP, Hansen MR, Al Dakhail AAA, House JW. Stapedectomy versus stapedotomy: comparison of results with long-term follow-up. Laryngoscope. 2002;112:2046-50.

6. Ruckenstein MJ, Nicolli EA. Is there a "best" stapes prosthesis? Laryngoscope. 2012;122:2123-4.

7. Huber AM, Hornung J, Plontke S, Röösli C, Iro H, Strutz J, Rahne T, Pezier TF, Kwok P. NiTiBOND an optimized selfcrimping stapes prosthesis for treatment of otosclerosis. Laryngorhinootologie. 2014;93:178-85.

8. Fang L, Lin H, Zhang T-Y, Tan J. Laser versus non-laser stapedotomy in otosclerosis: a systematic review and meta-analysis. Auris Nasus Larynx. 2014;41:337-42.

9. Brkic FF, Erovic BM, Onoprienko A, Janik S, Riss D, Lill C, Grasl S, Hamzavi J-S, Vyskocil E. Impact of surgeons' experience and the single-shot perioperative antibiotic prophylaxis on outcome in stapedotomy. PLoS One 2021;16:e0247451.

10. Yung MW, Oates J, Vowler SL. The learning curve in stapes surgery and its implication to training. Laryngoscope. 2006;116:67-71.

11. Ito T, Kubota T, Furukawa T, Matsui H, Futai K, Kakehata S. Transcanal endoscopic ear surgery for congenital middle ear anomalies. Otol Neurotol. 2019;40:1299-305.

12. Fang L, Xu J, Wang W, Huang Y. Would endoscopic surgery be the gold standard for stapes surgery in the future? A systematic review and meta-analysis. Eur Arch Otorhinolaryngol. 2021;278:925-32.

13. Nikolaos T, Aikaterini T, Dimitrios D, Sarantis B, John G, Eleana T, Konstantinos M. Does endoscopic stapedotomy increase hearing restoration rates comparing to microscopic? A systematic review and meta-analysis. Eur Arch Otorhinolaryngol. 2018;275:2905-13.

14. Ho S, Patel P, Ballard D, Rosenfeld R, Chandrasekhar S. Systematic review and meta-analysis of endoscopic vs microscopic stapes surgery for stapes fixation. Otolaryngol Head Neck Surg. 2021;194599821990669.

15.• Manna S, Kaul VF, Gray ML, Wanna GB. Endoscopic versus microscopic middle ear surgery: a meta-analysis of outcomes following tympanoplasty and stapes surgery. Otol Neurotol. 2019;40:983-993. This meta-analysis reports evidence supporting theapplication of endoscopes for tympanoplasty and stapes surgery.

16. Schmid P, Häusler R. Revision stapedectomy: an analysis of 201 operations. Otol Neurotol. 2009;30:1092-100.

17. Laske RD, Röösli C, Chatzimichalis MV, Sim JH, Huber AM. The influence of prosthesis diameter in stapes surgery: a metaanalysis and systematic review of the literature. Otol Neurotol. 2011;32:520-8.

18. Bonali M, Fermi M, Alicandri-Ciufelli M, Mattioli F, Villari D, Presutti L, Anschuetz L. Correlation of radiologic versus endoscopic visualization of the middle ear: implications for endoscopic ear surgery. Otol Neurotol. 2020;41:e1122-7.

19. Anschuetz L, Bonali M, Guarino P, Fabbri FB, Alicandri-Ciufelli M, Villari D, Caversaccio M, Presutti L. Management of bleeding in exclusive endoscopic ear surgery: pilot clinical experience. Otolaryngol Head Neck Surg. 2017;157:700-6.

20. Okuda H, Okamoto J, Takumi Y, Kakehata S, Muragaki Y. The iArmS robotic armrest prolongs endoscope lens-wiping intervals in endoscopic sinus surgery. Surg Innov. 2020;27:515-22.

21. Vittoria S, Lahlou G, Torres R, Daoudi H, Mosnier I, Mazalaigue S, Ferrary E, Nguyen Y, Sterkers O. Robot-based assistance in middle ear surgery and cochlear implantation: first clinical report. Eur Arch Otorhinolaryngol. 2021;278:77-85. This paper reports an in-depth description and clinical application of robot-assisted stapes surgery. 
22. Molinari G, Ragonesi T, Hool S-L, Mantokoudis G, Presutti L, Caversaccio M, Anschuetz L. Surgical implications of 3D vs 2D endoscopic ear surgery: a case-control study. Eur Arch Otorhinolaryngol. 2020;277:3323-30.

23. Doğan S, Bayraktar C. Endoscopic tympanoplasty: learning curve for a surgeon already trained in microscopic tympanoplasty. Eur Arch Otorhinolaryngol. 2017;274:1853-8.

24. Beckmann S, Yacoub A, Fernandez IJ, Niederhauser L, Fermi M, Caversaccio M, Bonali M, Anschuetz L. Exclusive endoscopic laser-stapedotomy: feasibility of an ovine training model. Otol Neurotol. 2021;42:994-1000.

25. Fernandez IJ, Bonali M, Yacoub A, Ghirelli M, Fermi M, Presutti L, Caversaccio M, Anschuetz L. Training model for salvage procedures in endoscopic stapes surgery. Eur Arch Otorhinolaryngol. 2021;278:987-95.

26. Anschuetz L, Bonali M, Ghirelli M, Mattioli F, Villari D, Caversaccio M, Presutti L. An ovine model for exclusive endoscopic ear surgery. JAMA Otolaryngol Head Neck Surg. 2017;143:247-52.

27. Rothbaum DL, Roy J, Stoianovici D, Berkelman P, Hager GD, Taylor RH, Whitcomb LL, Francis HW, Niparko JK. Robotassisted stapedotomy: micropick fenestration of the stapes footplate. Otolaryngol Head Neck Surg. 2002;127:417-26.

28. Vendrametto T, McAfee JS, Hirsch BE, Riviere CN, Ferrigno G, De Momi E. Robot assisted stapedotomy ex vivo with an active handheld instrument. Annu Int Conf IEEE Eng Med Biol Soc. 2015;2015:4879-82.

29. Razavi CR, Wilkening PR, Yin R, Lamaison N, Taylor RH, Carey JP, Creighton FX. Applied force during piston prosthesis placement in a 3D-printed model: freehand vs robot-assisted techniques. Otolaryngol Head Neck Surg. 2019;160:320-5.

30. Miroir M, Nguyen Y, Szewczyk J, Sterkers O, Bozorg Grayeli A. Design, kinematic optimization, and evaluation of a teleoperated system for middle ear microsurgery. Sci World J. 2012;2012:907372.

31. Peschka A, Berger T, Maier T, Scholz M, Lüth TC, Strauß G. Evaluation of a micro-manipulator for middle ear surgery: a preclinical trial. Laryngorhinootologie. 2016;95:112-7.

32. Strauss G, Maier T, Krinninger M, Berger T, Dietz A, Lüth T. Clinical use of a micromanipulator system: preliminary clinical experience in middle ear surgery. HNO. 2012;60:807-13.

33. Caversaccio M, Wimmer W, Anso J, et al. Robotic middle ear access for cochlear implantation: first in man. PLoS One. 2019; 14:e220543.

34. Koukkoullis A, Tóth I, Gede N, et al. Endoscopic versus microscopic stapes surgery outcomes: a meta-analysis and systematic review. Laryngoscope. 2020;130:2019-27.

35. Yasin R, O'Connell BP, Yu H, Hunter JB, Wanna GB, Rivas A, Simaan N. Steerable robot-assisted micromanipulation in the middle ear: preliminary feasibility evaluation. Otol Neurotol. 2017;38:290-5.

36. Bell B, Stankowski S, Moser B, Oliva V, Stieger C, Nolte L-P, Caversaccio $\mathrm{M}$, Weber $\mathrm{S}$. Integrating optical fiber force sensors into microforceps for ORL microsurgery. Annu Int Conf IEEE Eng Med Biol Soc. 2010;2010:1848-51.

37. Runge A, Hofer M, Dittrich E, Neumuth T, Haase R, Strauss M, Dietz A, Lüth T, Strauss G. Manual accuracy in comparison with a miniature master slave device-preclinical evaluation for ear surgery. Stud Health Technol Inform. 2011;163:524-30.

38. Brett PN, Baker DA, Reyes L, Blanshard J. An automatic technique for micro-drilling a stapedotomy in the flexible stapes footplate. Proc Inst Mech Eng H. 1995;209:255-62.

Publisher's Note Springer Nature remains neutral with regard to jurisdictional claims in published maps and institutional affiliations. 